\title{
Carnets
}

Revue électronique d'études françaises de l'APEF

Première Série - 4 | 2012

(Res)sources de l'extravagance

\section{À propos de l'extravagance dans Le Baiser (1925) de Pablo Picasso}

\section{Lydie Royer}

\section{(2) OpenEdition}

\section{Journals}

\section{Édition électronique}

URL : http://journals.openedition.org/carnets/6868

DOI : 10.4000/carnets.6868

ISSN : 1646-7698

Éditeur

APEF

\section{Édition imprimée}

Date de publication : 1 janvier 2012

Pagination : 147-165

\section{Référence électronique}

Lydie Royer, «À propos de l'extravagance dans Le Baiser (1925) de Pablo Picasso », Carnets [En ligne], Première Série - 4 | 2012, mis en ligne le 20 juin 2018, consulté le 03 mai 2019. URL : http:// journals.openedition.org/carnets/6868; DOI : 10.4000/carnets.6868

\section{(c) $(1) \Theta$}

Carnets est mis à disposition selon les termes de la licence Creative Commons - Atribution - Pas d'utilisation commerciale 4.0 International. 


\title{
À PROPOS DE L'EXTRAVAGANCE DANS LE BAISER (1925) DE PABLO PICASSO
}

LYDIE ROYER

Université de Reims

lydie.royer@univ-reims.fr

\section{Résumé}

Cet article propose d'étudier comment le tableau de Picasso, Le baiser, peint en 1925, aide ainsi à mieux saisir l'interprétation et l'effet de la notion de l'extravagance qui fonde, dans la société moderne, notre relation au monde et à l'Autre. L'article analyse la confrontation entre deux érotismes, masculin et féminin, selon trois axes, psychanalytique, artistique et philosophique: les théories de Sigmund Freud soulignent que, dans la relation érotique, l'homme occupe un rôle actif alors que la femme a un rôle passif. En revanche, la perspective des Surréalistes des années vingt est centrée sur l'aspect irrationnel du désir. Les théories de Carl Jung, de Georges Bataille y de Lacan, bien qu'elles soient différentes, tendent à démontrer comment l'être féminin aide l'être masculin à dépasser son angoisse pour mieux connaître l'Autre et soi-même dans la relation érotique.

\begin{abstract}
This article aims at studying how Picasso's work, Le baiser, painted in 1925, helps in understanding the interpretation and the effect of a notion of the extravagance, in the then modern society, based on our relationship with the world and with the Other. The article examines the confrontation between two eroticisms, male and female, from three points of view: psychoanalytical, artistic and philosophical, following the theories of Sigmund Freud, according to whom, in an erotic relationship, the male assumes an active role while the female, a passive one. On the other hand, the surreal perspective of the twenties is more focused on the irrationality of desire. The theories of Carl Jung, Georges Bataille and Lacan, although different, tend to show how the female helps the male overcoming his anxiety of how to better know the Other and himself in the erotic relationship.
\end{abstract}

Mots-clés: Picasso, Le Baiser, désir, corps, angoisse, érotisme, extravagance, art surréaliste Keywords: Picasso, Le baiser, desire, body, anxiety, eroticism, extravagance, surrealist art 
Pour le baiser, nos narines et nos yeux sont aussi mal placés que nos lèvres mal faites

Marcel Proust (1921)

Lieu commun de la littérature érotique depuis la Renaissance, le baiser, par ses significations symboliques liées à l'amour et à l'union sexuelle, a été également un motif artistique incontournable. En effet, les corps d'amants nus, tendrement enlacés, qui s'embrassent avec ardeur, célèbrent ce thème tant dans les fresques murales de la culture romaine que dans nombreux tableaux et sculptures ${ }^{1}$ jusqu'au $\mathrm{XX}{ }^{\mathrm{e}}$ siècle, comme Le Baiser de Gustav Klimt, peint en 1907.

Cependant, beaucoup d'artistes ont bouleversé les canons esthétiques, sans doute parce que le baiser, prémisse d'une force pulsionnelle et érotique, peut être représenté de manière extravagante, c'est-à-dire délirante, irrationnelle, hors norme et contraire aux convenances. Le Baiser de Picasso, par exemple, ne montre pas l'étreinte intime entre deux amants et permet ainsi de repenser des notions modernes comme l'extravagance qui fondent notre rapport au monde. II représente dans un premier temps le conflit fondamental freudien entre la pulsion de vie, Eros, et la pulsion de mort, Thanatos, entre raison et folie, amour et haine, répulsion et attraction.

L'expression formelle du visage de la femme conduit à une autre approche de l'extravagance comme déraison ou passion excessive et renvoie aux idées du Manifeste de Breton et des Surréalistes, selon qui, l'amour n'est pas la beauté de la femme, mais la transformation qu'il provoque sur celle-ci. Cette métamorphose de formes s'articule avec l'esthétique du Surréalisme, en vogue dans les années vingt, après la période du Cubisme analytique, qui avait pour but de dépasser le visible pour atteindre l'absolu et l'inconnu.

En outre, ce qui est extravagant pour le spectateur ne l'est pas forcément pour l'artiste. Aussi, l'étude des organes de la vue disséminés dans ce tableau, réels et symboliques, semble enrichir le sens du regard que porte le peintre sur le rapport de l'homme au monde et les rapports féconds entre l'art, la vie et le désir.

\section{La représentation de l'extravagance du désir masculin}

Ce tableau n'a pas la signification du baiser précédant l'acte sexuel ou l'amour; il n'est ni geste de dénouement heureux dans les belles histoires de princesses ou de contes de fée, ni simple motif de réconciliation entre l'homme et la femme dans la vie commune. Peint huit ans après Les Demoiselles d'Avignon qui avait fait scandale lors de sa première

\footnotetext{
${ }^{1}$ À part la sculpture d'Alex Boucher, (Hercule et Omphale,1735), les autres œuvres d'Auguste Rodin, 1888, de Constantin Brancusi,1908, et le tableau de Gustav Klimt, 1907, sont tous intitulés: Le Baiser.
} 
exposition, le titre annonce les préliminaires d'une relation intime mais représente davantage un conflit entre deux êtres; il devient source de questionnements sur la réalité de l'amour dans le couple. Les images représentent les rôles attribués aux deux sexes selon les théories de Freud dans l'acte d'amour, le passif pour la femme et l'actif pour l'homme.

Le baiser qui est habituellement promesse d'une union intime, comme chez les personnages de Shakespeare, Roméo et Juliette, ou mouvement d'un corps à la recherche de l'autre partenaire, devient chez Picasso, un rejet par la femme qui semble se défendre contre le désir agressif de l'homme. Picasso n'imite pas Le Baiser, œuvre emblématique de la période dorée du peintre autrichien Klimt, caractérisée par une surface picturale aux nombreuses feuilles d'or et inspirée des mosaïques byzantines. Klimt représente, en effet, la fusion des amants isolés du monde, dans une sphère libérée du temps et de l'espace, avec une femme agenouillée qui se donne, les yeux fermés, à son compagnon sur un tapis de fleurs et se laisse aller à la passion amoureuse; le plaisir amoureux semble être assimilé à la joie artistique.

Au contraire, dans Le Baiser de Picasso peint dix-huit ans après, l'image des deux amants autour de ce qui est censé les rapprocher, provoque et choque le spectateur: l'homme est à la recherche du sexe alors que la femme, en quête d'amour, y attache moins d'importance qu'au baiser lui-même. 


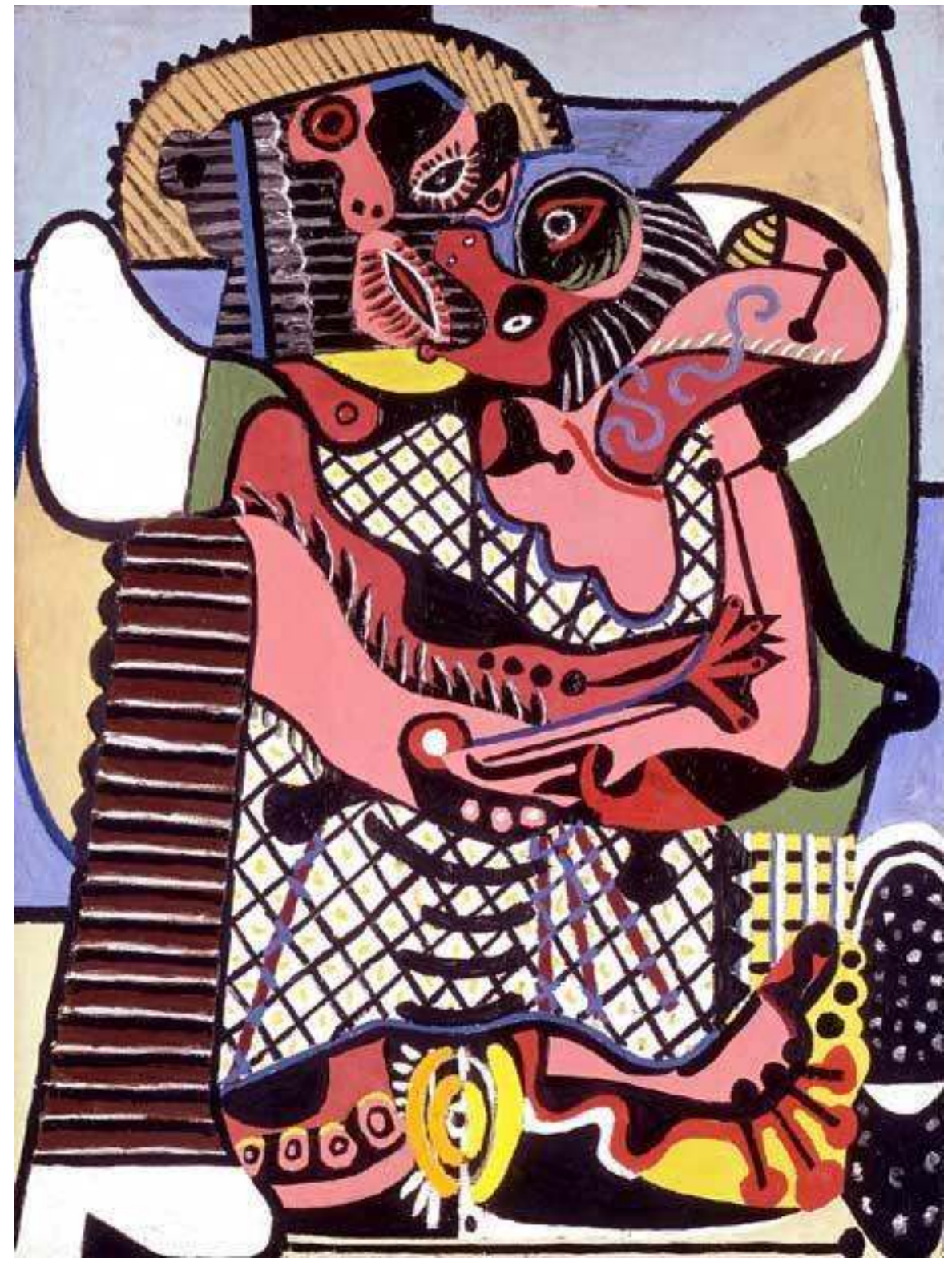

Pablo Picasso, Le Baiser, 1925, Musée National Picasso, Paris, 130 x 97,7 (c) Succession Picasso 2011

L'homme pointe son nez en forme de pénis dans la bouche de la femme, qui attrape un vagin. C'est cette image de la pénétration sexuelle ou du désir sexuel que la femme refuse et qui illustre l'approche freudienne du plaisir privilégiant une pulsion sexuelle essentiellement masculine. Les corps rapprochés créent un rapport de force et l'amour devient une lutte, un combat. L'extravagance du désir est l'état d'excitation visible chez I'homme: celui-ci est plus actif et a la position supérieure. Assis à gauche, et en proie à un dérèglement passionnel, il étouffe la femme en l'enveloppant fermement de son long bras hérissé de poils. La tension érotique de son corps trépidant, de son pied aux doigts écartés 
et de son nez en forme de pénis en érection, traduisent la violence de la pulsion intense qui l'habite.

La ligne droite de sa colonne vertébrale démontre qu'il l'écrase dans sa frénésie au point d'atteindre l'orgasme. La femme, contractée, ne ressent pas l'amour de l'autre et n'a d'autre choix que de refuser à l'homme, son vagin, l'objet maudit dont il veut s'emparer. Elle ne dévore pas un pénis mais un vagin, orifice et métaphore d'une bouche originelle, donc deux bouches, se présentant à l'homme, plus grand, qui est dans l'attente d'une pénétration et d'une fellation, et ne cherchant qu'à assouvir son appétit sexuel. Ainsi, l'acte sexuel n'est pas le but recherché par la femme qui veut que l'homme soit amoureux, et fixe son désir d'abord sur elle.

Dans ce tableau, la ligne des regards dessine une trajectoire qui converge directement vers le vagin. Mais les nombreuses autres lignes verticales et horizontales dessinent une esthétique dramatique de la relation sexuelle dans le couple. Les deux personnages incarnent Eros et Thanatos, qui symbolisent le thème du conflit dans la relation amoureuse. En effet, le baiser, prémisse érotique n'est pas perçu de la même façon pour les deux sexes car "avec le baiser, l'homme découvre l'haleine mais pour la femme, le baiser est la première des modalités qui consiste à offrir quelque chose de son propre corps; c'est le premier geste pour goûter au corps de l'homme" (Alberoni, 1990).

On connaît le rapport de Picasso au sexe, à la femme, à la nudité, au corps, ainsi que ses prouesses esthétiques liées aux organes phalliques et au constant dédoublement de la figure humaine. La présence des formes contorsionnées, obliques, la répétition d'organes en pointe, le nez, le pénis, le pied, les formes ovales entourées de poils hérissés, le vagin, les yeux, tous symboles sexuels, mettent en scène la puissante érection du mâle et l'hystérie de la femme.

Picasso semble, en effet, interroger de manière ironique la théorie freudienne de la sexualité féminine qui serait essentiellement clitoridienne, sans intervention du vagin et qui identifie le masculin et le féminin par deux termes antagonistes, le passif et l'actif.

Dans ce tableau, l'image est extravagante car elle illustre une femme dévoreuse qui théâtralise ses pulsions enfouies. L'image est à la fois une réelle allégorie de l'érotisme et de la sexualité et une illustration de la théorie freudienne de l'inconscient.

Selon Freud, l'inconscient de la femme est un surmoi sadique ou "un territoire étranger interne" alors que l'inconscient de l'homme est tourné vers les bons plaisirs de l'amour. Le baiser dévorateur du vagin donne à la femme l'apparence d'une mante religieuse. Les identités paraissent bien définies dans la relation homme-femme et l'élément masculin voit son Autre, le sexe féminin, avec malveillance, comme un être irrationnel et cruel prêt à dévorer son intimité. La femme serait l'incarnation du Mal et de la méchanceté selon le regard complexe que porte l'homme sur la bouche prête à avaler le vagin velu. En 
rejetant l'autre dans l'acte d'amour, elle exprime un repli sur soi et le refus de partager son corps. Elle dévorerait ainsi son propre vagin pour comme protéger ce dont l'autre veut s'emparer; elle est représentée comme une ogresse, une femme frigide, fermée qui se refuse au plaisir du mâle.

Ce tableau fait, semble-t-il, allusion à la vie conjugale du peintre à cette époque. Après la période du Cubisme, Picasso se marie en 1918 avec Olga, une danseuse des Ballets russes, femme bourgeoise d'un soldat de l'armée impériale qui renouvelle l'inspiration du peintre pour le corps féminin. Picasso peindra plusieurs portraits d'Olga; ils eurent un enfant, Paul. Mais au bout de dix ans, cette inspiration s'affaiblit. Olga s'ennuie et ne partage pas sa passion pour la peinture. Picasso étouffe dans ce cadre trop familial, il ne la peint jamais nue ni gaie, mais plutôt boudeuse et renfermée. Dans les portraits, Olga ne ressemble pas aux femmes suivantes de Picasso telles Marie-Thérèse, l'amour fou ou Dora Maar, l'amour passion. La crise personnelle que traverse le peintre à cette époque, après avoir connu une vie bien rangée et suite aux bouleversements esthétiques de l'époque et aux réactions négatives du public contre certains de ses tableaux, semble être à l'origine de la quête de nouvelles recherches plastiques qui le rapprocheront de l'univers des Surréalistes.

L'absence de communication est illustrée par les petites fragmentations éparses du tableau: les sexes, les yeux qui rappellent les bouches et l'anus entouré de poils, comme si l'un était en train d'épier l'autre, dans son désir et ses frustrations. Cette représentation de la femme n'est pas une nouveauté chez Picasso. La peinture et la littérature européenne du $X X^{e}$ que Picasso connaît, abondent en femmes irrésistibles, impitoyables, femmes fatales représentées souvent par des monstres mythologiques transformés en créature de chair (gorgones ou sorcières escortant Salomé, créature biblique et motif féminin lié à la mythologie du Mal) triomphant froidement de leurs victimes. En outre, le Mal ou le péché est assimilé à l'éternel féminin depuis la Bible. Dans cette représentation du $X X^{e}$ siècle, Picasso reprend pour mieux s'en distancier, l'image de la femme-objet, l'un des thèmes majeurs de Klimt qui montre, dans plusieurs de ses dessins, des femmes nues ou à demi-nues, allongées sur des divans ou des lits, offrant leurs corps aux spectateurs.

La scène du Baiser permet de lire le rapport de Picasso aux femmes: tantôt il les cajole, tantôt il les rejette. La tête de la femme, sorte de pieuvre qui attaque l'homme, renvoie à la description faite par Freud en 1922 de la Tête de Méduse peint par Caravage comme l'organe génital féminin entouré d'une chevelure à poils. Cette image a un effet repoussant et illustre l'interdit sexuel pour l'homme qui semble vouloir protéger son pénis. Celui-ci veut le plaisir érotique et non la souffrance. Pourtant l'exagération des couleurs rouge/rose (couleur des pulsions et des humeurs), renforce la déformation expressive du 
visage et fait naître l'émotion du spectateur qui a besoin de comprendre ce besoin de Picasso d'investir le personnage féminin de ce pathos irrésistible.

Incarne t-elle seulement la victime ou l'agressivité ou l'expression d'un désir sexuel irrationnel? Le détail extravagant des pointes noires en forme de sein ou de pénis et les écartèlements des membres de la femme ne démontrent-il pas que le corps échappe au contrôle de la raison? Des questions qui serviront de fil conducteur à l'analyse des formes et à l'étude de la figure féminine dans l'acte d'amour, selon un autre point de vue.

\section{La déformation délirante du corps féminin}

Picasso aimant subvertir les formes, soumet la figure féminine à un traitement qui transforme radicalement celle-ci, l'associant à des formes qui symbolisent des phallus en érection, sorte de virilité démiurgique de la création. La pulsion hystérique exprime de façon extravagante la modification du "moi" féminin dans le couple.

L'Etreinte, peint en 1903, montrait déjà une conscience claire chez le peintre de sa préoccupation artistique pour ce type de relation, faite souvent de conflits permanents même inavoués au sein du couple.

Dans La Danse, peint aussi en 1925, la présence bouleversante des trois corps féminins anguleux, lacérés, en mouvements syncopés avec une femme inclinée, à la tête renversée et une autre, à la jambe relevée, illustre l'affirmation d'une tension intérieure assez violente que le peintre répète dans Le Baiser. 




Pablo Picasso, La Danse, 215 x 142, Londres, Tates Gallery (c) Succession Picasso 2011

L'érotisme, sujet de prédilection du peintre, lui permet d'illustrer la complexité du désir dans le couple. L'analyse du comportement sexuel du couple aide ainsi à dépasser le seul point de vue de l'homme dans son élan vital vers la femme, pour considérer l'attitude de la femme, porteuse de n'importe quelle pulsion, telle l'agressivité, le défoulement, la volonté de dominer et aussi la propension à se différencier de l'Autre ${ }^{2}$.

Ainsi, la femme cesse d'être perçue comme une victime passive ou une incarnation du Mal et son visage tend à exprimer une forte intériorité et plutôt un désir secret, dans la relation sexuelle, différent de celui de son partenaire.

$\mathrm{Vu}$ sous cet angle, le spectateur a une autre perception de l'attitude en apparence extravagante de la femme dans sa relation érotique avec l'autre sexe.

La femme, qui, pendant des siècles, était en position soumise, avait accepté sa différence face à l'homme, mais au début du $\mathrm{XX}{ }^{\mathrm{e}}$ siècle, Picasso dans la période explosive

\footnotetext{
${ }^{2}$ Le refoulement par l'homme de ses tendances et de ses traits féminins détermine naturellement l'accumulation de ces besoins et de leurs exigences dans l'inconscient.C.G.Jung, Dialectique du moi et de l'inconscient, p.144.
} 
et surréaliste de sa peinture, choisit de montrer le contraire de cette soumission, c'est-à-dire, une sorte de résistance à l'oppression machiste en libérant la femme de ses pensées figées.

Dans Le Baiser, l'imagination est intarissable et représente une inspiration convulsive chère aux surréalistes: le fait d'exhiber le coté le plus intime de l'être féminin dans sa propre bouche constitue un acte impudique ou extravagant. La femme exprime son désir violent qui fait peur à l'homme. Et cette image marque, aussi bien la rupture avec le Cubisme et le Néoclassicisme que l'audace de Picasso qui s'engage plus nettement dans une nouvelle phase d'exploration de sa peinture. L'image met en exergue sous des traits saillants, le jaillissement d'une énergie primitive. Les lignes géométriques et la couleur rouge, plus agressive, traduisent l'hystérie d'une femme victime; elles exhibent aussi la gueule ouverte d'une femme visualisant son désir au sens de jouir, hurler, crier et cette scène dramatique et théâtrale du tableau nécessite l'exploration de formes nouvelles pour la représentation du corps féminin et la sexualité. La femme hystérique veut se défendre du pouvoir masculin; elle semble crier des désirs secrets dont elle a perdu la conscience claire. Ainsi, l'extravagance n'est pas dans la contemplation car l'intention du peintre n'est pas de créer la beauté mais de rendre compte d'une force pure, d'un pouvoir et d'une énergie. L'emploi des formes striées, distordues, mises au service de la libération de l'inconscient, fait surgir, en même temps, la crise intérieure, laquelle, était déjà le moteur des Demoiselles d'Avignon; ce tableau inaugurant le Cubisme et que Picasso voulait intituler Le Bordel philosophique, offre un spectacle de danse érotique avec des filles assises, se tournant, levant les bras, cuisses ouvertes, yeux fixes et hagards. Bien que le Surréalisme fût à l'opposé du Cubisme, Picasso fut attiré par les idées que ce mouvement revendiquait, telle l'émancipation récente de la femme. L'expression violente du visage féminin renvoie alors à Thanatos, pulsion de mort qui est nécessaire à l'existence de la pulsion de vie.

Toutes les transformations du corps illustrent la métamorphose du désir chez la femme avec des images qui heurtent la pudeur comme l'étrange tête de femme dont la bouche est remplacée par un pubis dans Le Viol représenté par René Magritte en 1934. 


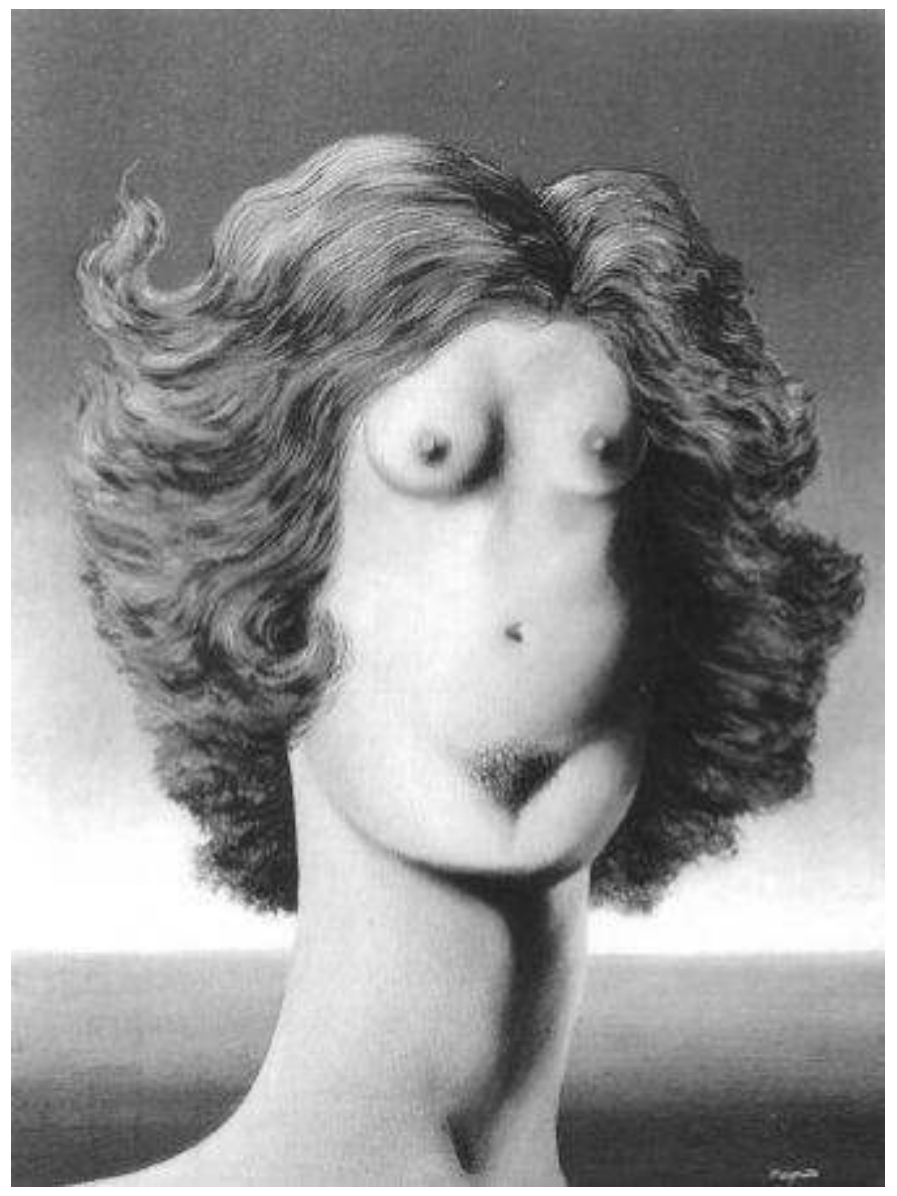

René Magritte, Le Viol, 1934

Huile sur toile, 25 X 18, Galerie, Isy Brachot, Bruxelles

Le cri muet qui semble secouer le personnage féminin de Picasso est d'autant plus saisissant qu'apparaissent les deux jambes écartées du corps étouffé qui se confondent avec ceux de l'homme. La déformation du corps féminin, emprisonné dans le bras massif du partenaire masculin et la bouche ouverte, sont deux signes d'une pensée irrationnelle qui rappelle un autre tableau de Picasso, le Grand nu féminin peint en 1929 avec une femme à la gueule hurlante, assise dans un fauteuil et dont les membres sont démesurément allongés et déplacés. Dans le Manifeste du Surréalisme de Breton publié en 1924, les rêves et l'inconscient ont une place privilégiée et permettent de dépasser le réel visible ou l'émotion créée par le regard. La mise en scène du comportement irrationnel du personnage féminin visualise à la fois l'émotion et la pensée. Les déformations du corps font surgir le cri qui est l'extériorisation d'un flux d'énergie longtemps retenu et qui rend compte des sexualités problématiques liées à la morale de la société bourgeoise et conformiste du début du $X X^{\mathrm{e}}$ siècle. Ici, la femme à la bouche largement ouverte est considérée comme une menace ou une force dévorante qui dévoile paradoxalement l'angoisse et la peur de l'homme très connue dans les sociétés primitives. 
Les dents grinçantes, image de la sexualité dans la peinture surréaliste, libèrent le désir refoulé ou l'agressivité que l'inconscient masculin rejette. Chez les Surréalistes, à la fois dans la peinture et au cinéma tels les chefs-d'œuvre de Luis Buñuel et de Salvador Dalí, Un Chien andalou (1925) suivi de L'Âge d'or (1930), l'image, sorte de plongée dans les recoins mystérieux de l'inconscient, a toujours été déstabilisante. Les recherches constantes du peintre autour de la représentation du visage et de l'anatomie du corps constituent des expérimentations successives jusqu'à la mise en œuvre d'une nouvelle facette de son art.

Au delà d'une simple allusion à la crise conjugale entre Picasso et son épouse Olga, le tableau se fait l'écho de l'intérêt des Surréalistes pour le cannibalisme. Le Baiser n'est pas extravagant au sens commun du terme, c'est un chef d'œuvre de la métamorphose des formes traditionnelles et une manifestation des nouvelles possibilités d'expression. En ce sens, Le Baiser est une transgression des limites imposées par les normes de la société bourgeoise, pour une remise en question des comportements de chacun dans la relation sexuelle. II devient moment de tensions reproduit dans l'expérience esthétique grâce à la technique surréaliste; il crée l'extravagant et s'ouvre en même temps sur une nouvelle compréhension de la relation globale entre l'art et la sexualité. L'image extravagante, dans la peinture, n'a de sens que si elle choque la perception du spectateur forçant l'artiste à s'interroger continuellement.

Aussi l' extravagance est-elle une notion qui permet de revenir en arrière et peut donner lieu à une autre évaluation du même thème dans un contexte différent pouvant soit résoudre les interrogations posées, soit faire face aux réponses restées en suspens qui mêlent inlassablement angoisse et désir dans la relation sexuelle.

Ce tableau surréaliste se compose de tous les ressorts de l'extravagance composés de désir, de répulsion, d'angoisse, d'attirance, de névrose, de séduction, de perversité et d'interrogations. Se remettre en question sur les délires du passé, c'est aussi réviser les images premières qui avaient ébranlé les perspectives habituelles du spectateur.

En effet, Picasso a éprouvé, à l'âge de ses 88 ans, le besoin de représenter le même thème en gardant le même titre, à la fin des années soixante, mais sous une forme différente; en effet il ne reproduit que les têtes des personnages unis par un baiser de bouche à bouche, signe d'égalité et d'harmonie dans le couple. Le Baiser peint à Mougins en 1969 semble être une réponse à la forme du désir conflictuel du tableau de 1925, car il aplanit tous les doutes posés au milieu des années vingt sur la représentation de la violence du désir et de la sexualité entre deux amants. 


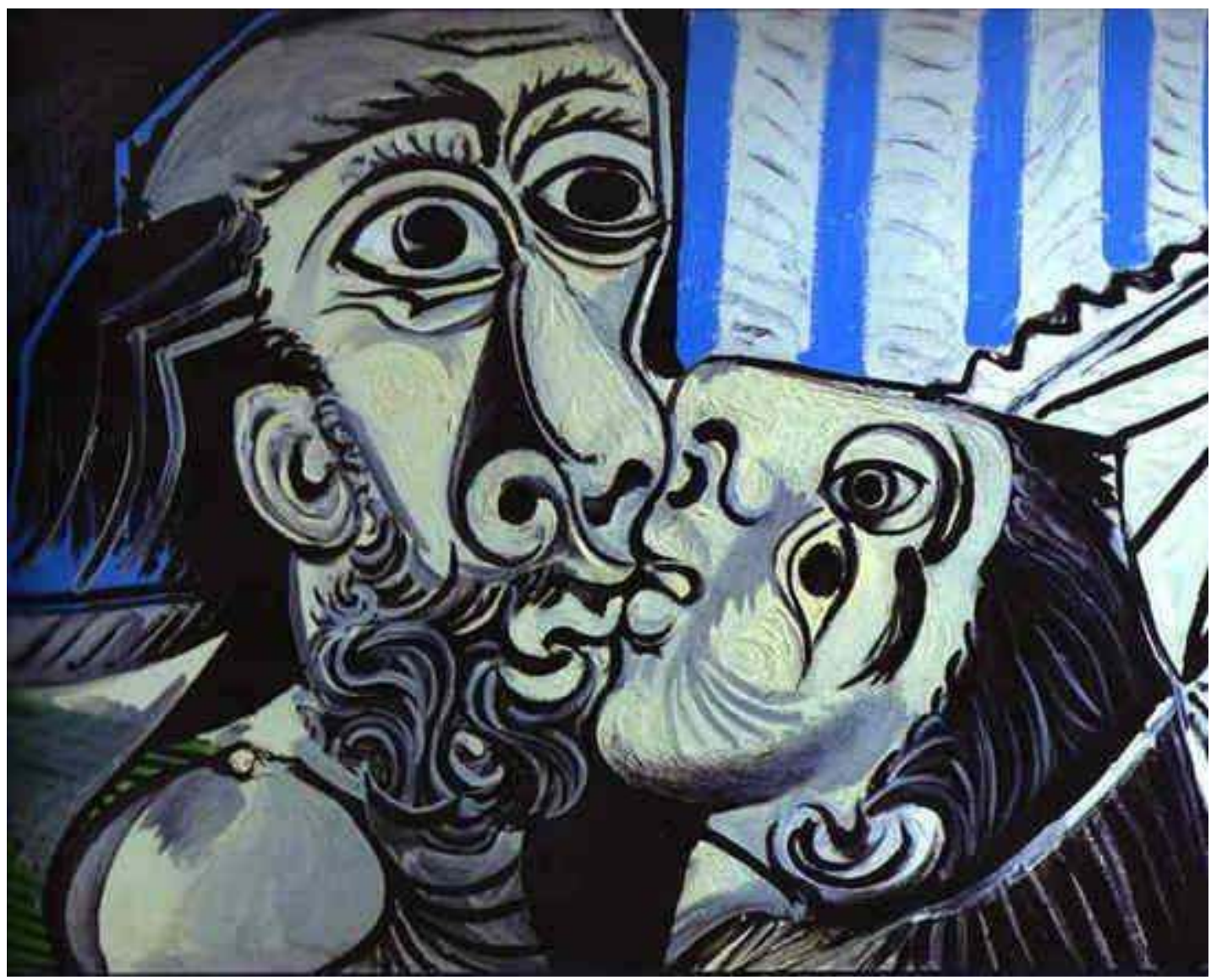

Picasso, Le Baiser, 1969, Musée Picasso, Paris, 97 × 130

(C) Succession Picasso 2011

Contrairement au tableau de 1925, leurs visages sont plus rapprochés et s'emboîtent jusqu'à ce que leurs nez se confondent en se prolongeant l'un dans l'autre et leurs bouches se mêlent dans d'infinis plis et replis. L'alliance des lignes courbes des organes sensuels du visage (nez, oreilles, yeux, bouches) visualise la tension esthétique qui entraîne l'ensemble de la composition dans un mouvement tourbillonnant où les différences s'annulent pour que se rapprochent les corps. II n'y a pas de transgression dans ce tableau mais on reconnaît le goût excessif de Picasso pour la déformation à l'extrême des visages pour mieux les nouer et exprimer la sensualité exacerbée du désir; l'ardeur du baiser, symbole de l'harmonie dans le couple est le signe de l'apaisement des tensions du passé dans le couple. Dans ce tableau, les thèmes et motifs sexuels des années précédentes ressurgissent comme une obsession chez le peintre, ce qui lui vaudra le surnom: "Picasso, le sage et le fou", "l'infatigable". Entre mai 1969 et février 1970, il peint une trentaine de baisers et de couples; certains, exposés au Palais des Papes à Avignon en 1970, ont fait scandale par leur caractère cru dénonçant les tabous persistants du siècle. Le Baiser de 1969 est plus figuratif mais devient plus serein et tendre; il reflète la nostalgie d'un vieillard de 90 ans qui pense à 
sa jeunesse; l'homme est barbu et chauve et la femme avec la tête en arrière et les cheveux longs retenus par une cordelette, rappelle certains détails de Guernica comme La femme qui pleure. La position perpendiculaire de ses yeux reste en contraste avec ceux de l'homme devenu plus serein et réaliste. L'étirement des formes dans les visages illustre le même climat de force et d'énergie qui caractérise toute la peinture de Picasso et l'obsession de la forme féminine pour signifier la fusion amoureuse. Le peintre utilise peu de couleurs. Les rayures blanches et bleues du fond sont les seules touches de lumière qui contrastent avec la grisaille qui domine l'ensemble; la chromatique froide tempère l'intensité du transport amoureux tandis que les amants semblent éternellement figés dans leur étreinte amoureuse. Le couple est uni, la femme devient désir et objet sexuel; son visage est identique à celui des autres représentations du même thème des années soixante où les femmes sont souvent regardées ou épiées par des vieillards concupiscents; le même désir pervers rejaillit avec, par exemple, La pisseuse (1965), sur la plage où le regard oriente davantage vers le sexe, le pubis, le ventre rond et les traits difformes du corps. Aussi, l'artiste intervient également pour libérer l'homme des contraintes morales "qui musèlent son imagination et frustrent ses désirs". (Claude Murcia, 1994).

Le tableau de 1969 semble répondre à l'extravagance du tableau de 1925 mais il renvoie à la fin des années soixante à la révolte générale des jeunes de l'époque contre les tabous et les mœurs de la morale bourgeoise autour de la sexualité.

Les yeux ouverts de l'homme dans ce tableau traduisent son émotion; son regard érotique exprime sa maturité dans la relation avec l'autre sexe; les lignes courbes sont en harmonie dans les deux têtes et dévoilent la passion physique entre les deux êtres. L'image de 1969 devient plus philosophique et semble résoudre le premier conflit entre Eros et Thanatos, entre pulsion de vie et pulsion de mort, dépassant ainsi le concept de Freud de la passivité féminine dans la sexualité. Ce couple est réuni par un baiser qui scelle toute la synthèse des motifs picturaux de l'union entre l'art et la vie. L'art permet de visualiser les comportements complexes des partenaires de chaque sexe en amour; il peut ainsi représenter les différents jeux de séduction et de l'amour à travers des images obsédantes et extravagantes exprimant la violence, le désir agressif, l'étreinte, la vie, la mort et la peur.

Cette réactivation du Baiser de 1925 réaffirme à nouveau la passion de Picasso pour la recherche formelle; peindre et posséder sont pour lui, les deux faces d'un même phénomène. L'art n'est jamais chaste; il est extravagant parce qu'il est l'expression formelle des pulsions vitales les plus intenses de l'être humain. 


\section{Des yeux partout pour voir et pour savoir}

Cette expression extravagante de la sexualité s'éclaircit dans les déplacements des organes et dans la dimension érotique du regard. Le désir cherche inlassablement à ouvrir l'œil du spectateur. La pulsion destructrice qui habite la femme est aussi le motif de la création qui s'extirpe du tableau. Aussi, la bouche ouverte n'est pas seulement contemplative ni fondée sur la seule représentation de l'hystérie ou du refoulement; mais elle devient recherche d'expression, langage et discours sur un vrai désir et la possibilité de présenter la femme dans sa bipolarité, à la fois dans sa sensibilité et sa spiritualité.

L'œil, à la fois organe de perception visuelle, symbole sexuel et instrument du désir approfondit la notion de l'extravagance.

L'œil de l'homme entouré de poils rappelant le vagin dans la bouche de la femme ne serait-il pas une sorte de mise à nu du personnage féminin?

L'œil gauche plus petit et discret du personnage masculin ne manifeste-t-il pas au contraire une distance entre l'homme voyeur et la femme objet du regard?

La distinction entre la forme des regards des deux personnages et les mises en abyme des organes de la vue, disséminés sur les corps, orientent la réflexion à la fois vers la réception de l'image par le spectateur et vers la conception de celle-ci par le peintre. Comment l'artiste travaille t-il la position des corps et des regards afin de représenter la complexité de la relation sexuelle dans le rapport à l'autre? Ces interrogations peuvent apporter quelques réponses sur le sens de la mise en scène du regard, une forme de transgression qui devient aussi un jeu créateur dans l'œuvre.

Les déplacements d'organes dont l'un substituant l'autre et les nombreuses rayures sur le corps définissent une conception esthétique de la fragmentation des formes qui permet d'inverser la perspective reçue et d'arracher le spectateur aux conventions de la perception du Baiser.

L'homme du tableau semble être celui à qui on a attribué plus d'organes liés à la vue et dont la plupart ont la forme de son œil droit comme pour combler un manque, un vide. Le premier regard de l'homme sur la femme est un regard de fierté et de supériorité masculine. "Voir, c'est d'abord voir qu'on est un sexe, c'est-à-dire un être séparé. Voir est un phénomène indissolublement lié au voyeurisme, c'est-à-dire à la fascination de la différence sexuelle."(PONTALIS, 2004). La position des têtes des deux personnages, le choix des différentes formes pour représenter leurs yeux démontrent que leurs regards ne se croisent pas; en effet, le regard vertical et asymétrique de l'homme est celui d'un "moi" qui regarde vers le bas et construit l'infériorité de la femme en la figeant dans une identité qui ne lui correspond pas. L'homme regarde, par une fente, la partie intime de la femme et observe le 
désir refoulé de celle-ci. Mais cette image a un pouvoir terrible et lui permet de penser ou de se voir lui-même dans la relation avec l'Autre.

Chez Picasso, il y a toujours une quête lancinante du désir et du plaisir et le jeu des regards est nécessaire à l'inspiration érotique; l'amour est un combat et habite toutes ses œuvres. Aussi, l'attitude de l'homme qui surveille la femme sans être vu, avec un regard jouissif, est une relation perverse, extravagante, qu'il imagine en prévoyant le mal qu'il va subir. Le pervers est d'abord celui qui jouit de la douleur qu'il subit sous le regard de l'Autre ou de celle qu'il inflige.

L'excentricité du corps féminin fragmenté révèle au contraire un désir d'autonomie et d'indépendance qui peut aussi être pervers. Picasso fait surgir l'étrangéité de l'autre sexe pour le désir et l'image du désir de la femme semble se rapprocher de la thèse de Lacan pour qui "le désir pervers est le désir vrai et qui pose que sa vérité consiste à rejoindre l'autre par le seul moyen possible: lui faire reconnaître violemment sa propre division." (PONTALIS, 1988). La pulsion perverse serait ici le seul accès à l'altérité, pour la femme. Ainsi, le regard n'est pas seulement vision de l'Autre comme objet, elle est aussi vision de l'Autre comme sujet pensant; la femme, attentive à son intimité, regarde vers le haut et construit aussi l'hégémonie de son moi pour sortir de cette position infligée.

L'extravagance s'explique également par le déplacement des organes sur le corps: la mise en rapport du vagin et de la bouche, l'alliance d'une même cavité, l'anus, pour un seul corps semblent répondre à la recherche artistique de lieux symboliques d'expulsion du désir. L'œil à la place de l'anus entouré de poils blancs, en bas, et attribué à un seul corps double unissant l'homme et la femme, renvoie à la perception surréaliste de la sexualité d'André Breton. Ainsi, le sexe et l'anus cessent d'être perçus contradictoirement et forment un seul organe bisexuel, chargé de brouiller les conflits apparents entre les sexes, afin de marquer davantage la coexistence conflictuelle en chaque individu.

La Volonté d'ouvrir l'œil du dedans, idée très chère à Georges Bataille, associe la jouissance à l'angoisse de la mort et conduit à avoir une vision globale de l'expérience masculine et féminine dans la relation sexuelle, une approche visant à la compréhension de l'altérité avec l'autre sexe. La femme est cet autre dont l'homme a besoin, pour se compléter et se dépasser. L'attitude de la femme face au désir de l'homme prend un autre sens, selon Carl Gustav Jung qui propose une autre dimension que celle de Freud, basée sur l'inconscient et sur le désir refoulé. À la différence de Freud, Jung développe une théorie de l'inconscient qui s'écarte des fondements physiologiques ou du dualisme entre les deux sexes et oppose aux termes de Freud, actif et passif, les concepts de féminin et masculin chez les deux êtres. Tout masculin est doté de trait féminin (anima) et tout féminin est doté du trait masculin, (animus). Jung précise que l'anima et l'animus se situent à la limite supérieure du clair-obscur de l'être. L'animus de la femme est source d'opinions et la rend 
aussi dynamique et autoritaire que l'homme. II est aussi, selon Jung, cet "être créateur", une matrice destinée à "faire fructifier le coté féminin de l'homme". La femme manifeste son individualité dans la sexualité et oppose l'altérité à l'homme, lui permettant de voir sa brutalité pour pouvoir mieux se libérer, une idée qui va à l'encontre de la pensée de Freud selon qui, la part du refoulé dans l'acte sexuel est toujours attribuée à l'élément féminin. Son cri est une sorte d'interrogation et renvoie à la volonté de savoir étudiée par Michel Foucault, qui, dans ses essais, Histoire de la sexualité, aide à mieux cerner l'alliance audacieuse entre les autres organes dans ce tableau, chargés de modifier le mode de perception habituel du spectateur.

À ce propos, Foucault a aussi étudié la mise en forme de l'expérience des plaisirs ou la manière dont un sujet se constitue dans son rapport avec son sexe. La femme représente l'autre absolu permettant à l'homme de se voir soi-même comme un autre.

Les délires de Picasso dans ce tableau avec les nombreuses formes d'orifices et de réceptacles traduisent non seulement un besoin de révéler la réalité de sa vie mais illustrent au moyen des déformations, la vitalité intérieure et la caractéristique du désir de chaque sexe. Le regard de chaque personnage traduit ainsi la phase d'angoisse qui les habite dans la quête d'une relation juste, entre la sauvegarde de leur indépendance et le désir de l'Autre. Voir c'est aussi savoir, c'est exprimer le désir de connaître le mystère et la vérité de l'être.

Mais en dehors de ces deux personnages, il y a un autre regard qui ne prend pas position, c'est celui de l'artiste qui renouvelle sans cesse son regard sur sa création. L'œil gauche en coin de l'homme voyeur, regarde aussi l'autre œil bordé de longs poils et similaire au vagin: un œil mis en abyme dans une fente ou une cavité qui constitue une transgression de la morale sexuelle et qui répond en même temps à l'ambiguïté de la relation entre contemplation artistique et érotisme. L'œil en coin qui observe le spectateur est l'organe de sens, capable de voir aussi l'Autre et permet d'accéder à la connaissance, c'est-à-dire de se confronter à celui de l'artiste en train de créer les images.

Et plus qu'un sexe, l'œil poilu est aussi celui de l'artiste qui pense, fixe et interroge le regard du spectateur. II est la métaphore d'une vision personnelle du monde intérieur de l'artiste confronté à ses interrogations et à la sexualité dans le couple. Le jeu instauré entre ces multiples regards crée l'opposition entre voir et désirer, entre regard érotique et regard esthétique qui est l'opposition entre art et vie. Picasso, conscient de cette opposition fondamentale de son époque entre l'art et la vie, crée, sous le mode de l'ironie, un nouvel érotisme destiné à changer la relation passionnelle entre homme et femme.

Rappelons que les yeux déformés traduisent aussi les transgressions des codes opérés par les Cubistes dans la décomposition des formes, exprimant aussi une libération du regard. 
L'œil recrée la dialectique du regard sur l'Autre, réfléchit la représentation des histoires et des antagonismes au sein du couple et pose la question de la dualité qui repose à l'intérieur de chaque sujet. Le Baiser, de par son extravagance, va au delà de l'émotion ou du désir sensuel et exige finalement un regard plus profond de l'Autre; il n'est pas seulement une scène de pulsions de l'un vers l'autre sexe mais bien le lien pulsionnel entre amour et art comme force créatrice, vie sexuelle féconde qui trouve toute sa symbolique dans la femme.

C'est une œuvre qui nous permet de nous regarder au dedans de nous-mêmes et de voir nos différences par rapport à l'Autre.

La représentation du Baiser par Picasso convoque l'extravagance mais illustre, avant tout, la frénésie créatrice qui s'accroît chez le peintre à mesure qu'il avance en âge. Ce thème est extravagant en ce sens qu'il traduit sur un plan philosophique, la relation de soi à l'Autre et sur le plan artistique, la possibilité de jouer avec les formes, bref, d'être à la fois sujet et objet de sa peinture.

Picasso rejoint ici Bataille, selon qui, la transgression est la condition et l'essence même de l'érotisme. L'association délirante des organes, le jeu des déplacements des symboles phalliques, contribuent à associer les contraires dans une scène intime; ils font jaillir les sentiments refoulés ou agressifs et les souvenirs douloureux à l'image de la créativité picturale effervescente du peintre, capable de représenter l'être humain dans toute son intériorité et ses désirs inavoués.

Le thème du baiser qui fut, à l'origine, motif de bouleversement des consciences, et très souvent repris par des Cubistes expressionnistes et par des Surréalistes, propose des images qui permettent d'apprécier de sérieux aspects de la notion de l'extravagance dans la société moderne. Le Baiser renoue également avec les images de la sexualité, présente dans Les Demoiselles d'Avignon, telles la perplexité, l'agressivité et la liberté et permet la réinvention formelle d'un motif artistique de toutes les époques. II symbolise l'érotisme et la volonté collective d'abolir la différence, entre les sexes, établie depuis la Genèse. Picasso représente la violence du désir masculin mais exalte aussi le désir féminin renvoyant aussi à Jung, pour qui "la rencontre amoureuse n'est bouleversante que lorsque l'être masculin côtoie une personne de l'autre sexe sur laquelle son propre anima peut se projeter". (JUNG, 1964). En outre, ce thème assez provocateur peint par Picasso en 1925 et réinventé en 1969, met en lumière combien l'art et ses extravagances peut illustrer le changement du rapport de l'homme à la femme en sollicitant le corps et le regard dans la représentation des formes surtout au début du vingtième siècle. Le couple, emporté par sa passion, s'entremêle, s'entrechoque et s'entredévore. En outre, ce thème trouve un écho dans les constantes interrogations de l'homme de plus en plus morose de notre société du XXI ${ }^{e}$ siècle et dont les désirs frustrés rappellent les mêmes débats des années 20/30 et les slogans des jeunes de 
1968 autour de la sexualité. La scène du Baiser regorge d'organes visuels et de symboles de la sexualité; elle illustre ainsi le thème de l'extravagance non seulement de manière émotive mais permet à l'artiste d'explorer toutes les possibilités formelles de l'objet: elle peut représenter aussi bien le combat que se livre Eros et Thanatos en l'homme, que la folie créative chez le peintre, pour qui, l'amour, l'art et la vie sont intrinsèquement liés. 


\section{Bibliographie}

AlBERONI, Francesco (1990). L'érotisme. Paris: Ramsay (traduction française).

BATAILlE, Georges (1961). Les larmes d'Eros. Paris: Jean Jacques Pauvert, coll.10/18.

BATAILLE, Georges (1957). La littérature et le mal. Paris: Gallimard.

COMAR, Philippe (1993). Les images du corps. Paris: Gallimard.

FrEUD, Sigmund (1905). Trois essais sur la théorie de la sexualité. Paris, Gallimard, 1987.

Maldonado, Guitemmie (2007). Lire la Peinture de Picasso. Paris: Larousse.

JUNG, Carl Gustav (1963). L'Ame et la Vie. Paris: Buchet/Castet (traduction française).

JUNG, Carl Gustav (1964). Dialectique du moi et de l'inconscient. Paris: Gallimard.

FOUCAULT, Michel (1976). Histoire de la sexualité, la volonté de savoir. Paris: Gallimard.

LACAN, Jacques (2004). La loi, le sujet et la jouissance. Paris: Michalon.

MURCIA, Claude (1994). Un chien andalou. L'Âge d'or, Paris: Nathan.

PONTALIS, Jean Bertrand (1964). Le Mal. Paris: Gallimard.

QUINODOZ, Jean (2004). Lire Freud. Paris: Puf. 